\title{
An Intelligent Genetic Algorithm for PAPR Reduction in a Multi-Carrier CDMA Wireless System
}

\author{
Yang Zhang ${ }^{1}$, Qiang $\mathrm{Ni}^{1}$, Hsiao-Hwa Chen ${ }^{2}$, Yonghua Song ${ }^{1}$ \\ ${ }^{1}$ Electronic \& Computer Engineering, School of Engineering and Design, Brunel University, West London, UK \\ ${ }^{2}$ Institute of Communications Engineering, National Sun Yat-Sen University, Taiwan \\ E-mail: \{yang.zhang, qiang.ni\}@brunel.ac.uk, hshwchen@ieee.org
}

\begin{abstract}
A novel intelligent genetic algorithm (GA), called Minimum Distance guided GA (MDGA) is proposed for peak-average-power ratio (PAPR) reduction based on partial transmit sequence (PTS) scheme in a synchronous Multi-Carrier Code Division Multiple Access (MC-CDMA) system. In contrast to traditional GA, our MDGA starts with a balanced ratio of exploration and exploitation which is maintained throughout the process. It introduces a novel replacement strategy which increases significantly the convergence rate and reduce dramatically computational complexity as compared to the conventional GA. The simulation results demonstrate that, if compared to the PAPR reduction schemes using exhaustive search and traditional GA, our scheme achieves $99.52 \%$ and $50+\%$ reduction in computational complexity respectively.
\end{abstract}

Index Terms - PAPR, MC-CDMA, Genetic Algorithm, Partial Transmit Sequence

\section{INTRODUCTION}

$\mathrm{M}$ ulti-Carrier Code Division Multiple Access (MC-CDMA) is an emerging multi-carrier broadband wireless transmission technique that combines the advantages of both Code Division Multiple Access (CDMA) and Orthogonal Frequency Division Multiplexing (OFDM) together [1-3]. In MC-CDMA, spreading chips of each user are modulated over all the available subcarriers achieving high frequency diversity gain. This allows all users to simultaneously share the same frequency band by transmitting data on all subcarriers at a same time. In an MC-CDMA system, multi-path fading can be mitigated effectively by using OFDM modulation. However, MC-CDMA also suffers from the peak-to-average power ratio (PAPR) problem because of the inherent nature of OFDM modulation. As the number of subcarriers increases, the hostile effect of high PAPR becomes more severe in an MC-CDMA system, which may cause spectrum regrowth, in-band distortion and impairment of detection efficiency when signal is passed through nonlinear devices used for system signal processing, such as digital-to-analog converters, mixers and transmit power amplifiers. Hence, reducing PAPR is a crucial research topic for next-generation MC-CDMA based broadband wireless systems.

Generally, there are four main categories of PAPR reduction schemes: clipping \& peak windowing [4-6], block coding [7], selected mapping (SLM) $[8,9]$ and partial transmit sequences (PTS) scheme [10]. It is known that PTS can effectively reduce the PAPR by increasing the number of sub-blocks without modifying the number of subcarriers. In PTS, the input data block is partitioned into a number of disjointed sub-blocks or namely clusters which are combined to minimize PAPR. The phase of each sub-block is rotated by a phase factor after applying Inverse Fast Fourier Transform (IFFT) in each sub-block. Then each resulting sub-block is superimposed. The summation sequence is measured regarding its PAPR and the resulting sequence with minimum PAPR is selected to be transmitted. Thus, as the number of sub-blocks increases, the PTS can mitigate the effect of PAPR effectively.

However, one main drawback of PTS scheme is its high computational complexity which is proportional to the number of sub-blocks and phase factors. There is some research work proposed [11-15] to reduce the computational complexity. However, to the best of our knowledge, either their computational complexity is still high, or the PAPR reduction performance is not good enough. In this paper, we propose a novel scheme called minimum-distance guided genetic algorithm [16] (MDGA) to dramatically reduce the computational complexity while achieving a good near-optimum performance close to the exhaustive search.

The rest of this paper is organized as follows: Section II presents a typical MC-CDMA model and PAPR is explained followed by a conventional exhaustive PTS solution. In Section III our novel MDGA is proposed for PTS with low computational complexity. Section IV compares our scheme with other schemes using simulations. Finally, we conclude the paper in Section V.

\section{EXHAUSTIVE PTS BASED PAPR REDUCTION IN AN MC-CDMA SYSTEM}

We consider a bit-synchronous MC-CDMA transmitter as illustrated in Fig. 1, where $K$ users simultaneously transmit data bits over an additive white Gaussian noise (AWGN) channel.

Without loss of generality, a typical MC-CDMA transmitter model is adopted. Each user bit is first spread by a unique pseudo-random sequence. Then each chip of the corresponding spread signal is modulated on each orthogonal subcarrier. Assuming that the number of chips is equal to the number of sub-carriers, the $k^{\text {th }}$ user's transmitted signal is given by, 


$$
\boldsymbol{s}_{k}(\boldsymbol{t})=\frac{1}{\sqrt{N}} \sum_{n=0}^{N-1} \boldsymbol{c}_{k, n} \cdot \boldsymbol{b}_{k} e^{j \omega_{n} t}, 0 \leq t \leq N \boldsymbol{T}_{c}
$$

where, $N$ : The number of subcarriers,

$b_{\mathrm{k}}$ : The transmitted bit of the $k^{\text {th }}$ user,

$c_{\mathrm{k}, \mathrm{n}}$ : The $n^{\text {th }}$ spreading chip of the $k^{\text {th }}$ user,

$\mathrm{S}_{\mathrm{k}}=\left\{\mathrm{c}_{\mathrm{k}, 0} \mathrm{~b}_{\mathrm{k}}, \mathrm{c}_{\mathrm{k}, 1} \mathrm{~b}_{\mathrm{k}}, \ldots, \mathrm{c}_{\mathrm{k}, \mathrm{N}-\mathrm{b}} \mathrm{b}_{\mathrm{k}}\right\}$ : The chip sequence of the $k^{\text {th }}$ user over all sub-carriers,
$T_{c}$ : The chip duration,

$\omega_{\mathrm{n}}=2 \pi \mathrm{f}_{\mathrm{n}}$, where $f_{n}=n \Delta f(\mathrm{n}=0, \ldots, \mathrm{N}-1)$ is the $\mathrm{n}^{\text {th }}$ subcarrier frequency and the frequency spacing is $\Delta f=1 / N T_{c}$.

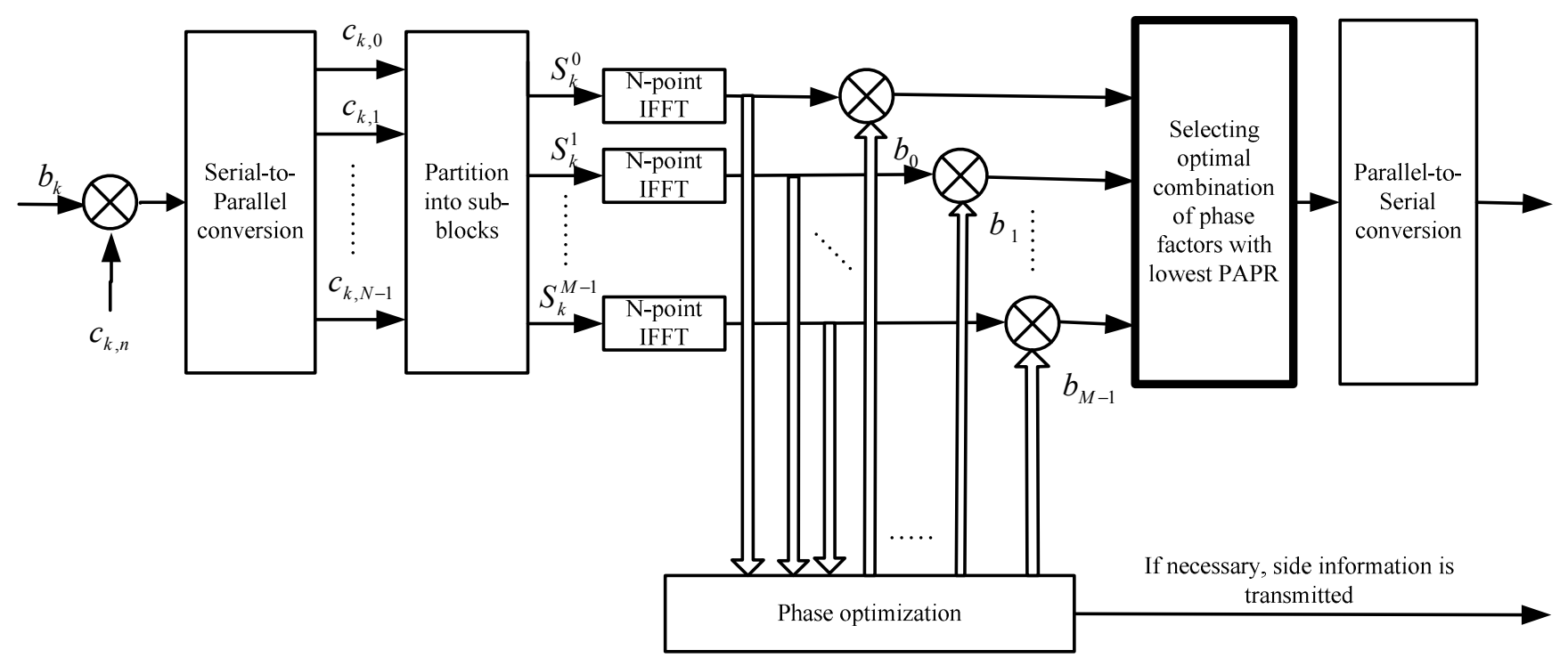

Fig. 1. The transmitter model of our MC-CDMA system

Therefore, the PAPR of the transmitted MC-CDMA signal in one symbol duration is defined as

$$
\operatorname{PAPR}\left(s_{k}(t)\right)=10 \log _{10} \frac{\max _{0 \leq t \leq N T_{c}}\left|s_{k}(t)\right|^{2}}{E\left[s_{k}(t)^{2}\right]}(\mathrm{dB})
$$

where $E[\cdot]$ denotes the expectation. Practically, the analog signal is usually sampled to become digital signal. Therefore, we can rewrite Equations (1) and (2) as

$$
S_{k} \stackrel{\Delta}{=} S_{k}\left(v T_{c}\right)=\frac{1}{\sqrt{N}} \sum_{n=0}^{N-1} S_{k} e^{j \omega_{n} v T_{c}},
$$

and

$$
\operatorname{PAPR}\left(s_{k}\right) \stackrel{\Delta}{=} \operatorname{PAPR}\left(s_{k}\left(v T_{c}\right)\right)=10 \log _{10} \frac{\max \left|s_{k}\right|^{2}}{E\left[s_{k}{ }^{2}\right]}
$$

respectively, where $\mathrm{v}=0,1, \ldots, \mathrm{N}-1$. The equation can then be normalized as

$$
\begin{aligned}
& S_{k} \stackrel{\Delta}{=} S_{k}(v)=\frac{1}{\sqrt{N}} \sum_{n=0}^{N-1} S_{k} e^{j \omega_{n} v} \\
& \quad=\operatorname{IFFT}_{N}\left(S_{k}\right)=\left[s_{k}(0), s_{k}(1), \ldots, s_{k}(N-1)\right]
\end{aligned}
$$

As shown in Fig. 1, the PTS scheme divides the input symbol block into $\mathrm{M}$ disjoint sub-blocks and multiplies them with a set of phase factors for phase rotation. The phase factors are given as $b_{w}=e^{j w \theta}$, where $w=0,1, \ldots, W-1, \theta=\frac{2 \pi}{W}$ and $W$ is a random integer. Each sub-block is transformed into partial transmit sequence using IFFT and then rotated by its allocated phase factor. Therefore the crucial objective is to optimally combine the sub-blocks and corresponding phase factors in order to transmit the superimposed symbol block with the lowest PAPR. Furthermore, in order to precisely estimate the PAPR of the true continuous modulated chip signal in MC-CDMA system, the chip sequence $S_{k}$ is oversampled [17] by means of padding $(L-1) \times N$ zeros, where $L$ is defined as an oversampling factor. Accordingly, $L N$-point IFFT is needed for each sub-block with $(L-1) N$ zero-padding. It is shown that in [8] oversampling factor equal or larger than 4 is sufficient to deal with the case. In this paper, the oversampling factor $L$ is chosen 4 . Therefore, by using oversampling, the process of PTS is formulated as:

$$
S_{k}=\sum_{m=0}^{M-1} S_{k}^{m}
$$




$$
\underline{\boldsymbol{s}_{k}}=\sum_{m=0}^{M-1}\left(\operatorname{IFFT}_{L N}\left(\boldsymbol{S}_{k}^{m}\right) \cdot \boldsymbol{b}_{w}\right)
$$

where $S_{k}^{m}$ denotes the $m^{\text {th }}$ sub-block and $\underline{s_{k}}$ represents the superimposed symbol block. Due to the linearity of discrete IFFT, we have

(8)

$$
\operatorname{IFFT}_{L N}\left(S_{k}\right)=\sum_{m=0}^{M-1} \operatorname{IFFT}_{L N}\left(S_{k}^{m}\right)
$$

As mentioned earlier, one significant drawback of the PTS based PAPR reduction scheme is the exhaustive iterative searching computational complexity, which is defined as $W^{M}$. The iterative flipping (IF) [8] is a simple method which can reduce the computational complexity to $(W-1) \times M$. However, its PAPR reduction performance is not very good. To deal with such a complex multi-objective optimization problem, genetic algorithm (GA) [16] might work. However, the computational complexity of traditional GA is still high. In this work, a novel minimum distance guided genetic algorithm (MDGA) is proposed to significantly reduce the computational complexity.

\section{Minimum Distance Guided Genetic Algorithm}

Our proposed novel GA is designed to accelerate the searching speed to find the optimal solution by adequately exploiting the output of the Iterative Flipping (IF) as a guidance. As compared to the conventional GA, we introduce a novel replacement strategy in both initial population matrix and mating pools. The idea is to keep a balanced search throughout all GA generations. Initial population matrix replacement allows us to start the initial search guided by the output of IF. Whereas, replacement of mating pools is an intelligent replacement strategy that plays a key role in providing quick convergence rate to our proposed GA. Combining the two schemes together offer our GA a good starting search point as well as increased convergence without introducing much complexity into the system. Our MDGA works as follows.

\section{A. Initial Population}

Considering that a good initial guess of the possible solutions is helpful for GA to obtain a good performance, the selection of initial population to get better initial estimate is created up by mutating the output of the iterative flipping (IF).

Let the total population of chromosomes be " $N_{p}$ ". In order to make sure that each phase factor of IF's output experiences change at least once, we generate the initial " $M$ " set of population by perturbing the IF's output in such a way that the Hamming Distance between IF's output and the new individual keeps "one", where $\mathrm{M}$ is the number of sub-blocks. The IF's output is considered as a good estimate. Additionally, to avoid occurrence of same parent pairs (incest) in initial population which degrade the searching diversity in the proposed GA, we assume $M<N_{p} \leq 2 M$. Yet, there is a high probability that a search which is only guided by IF's output according to Hamming Distance of one may get stuck at local optima with high PAPR values. In order to reduce this unwanted possibility and balance the trade-off between convergence rate and searching diversity, we make the rest of the population randomly mutated from the output of IF in order to explore other possible optimum solutions in the search space as shown in Fig. 2.

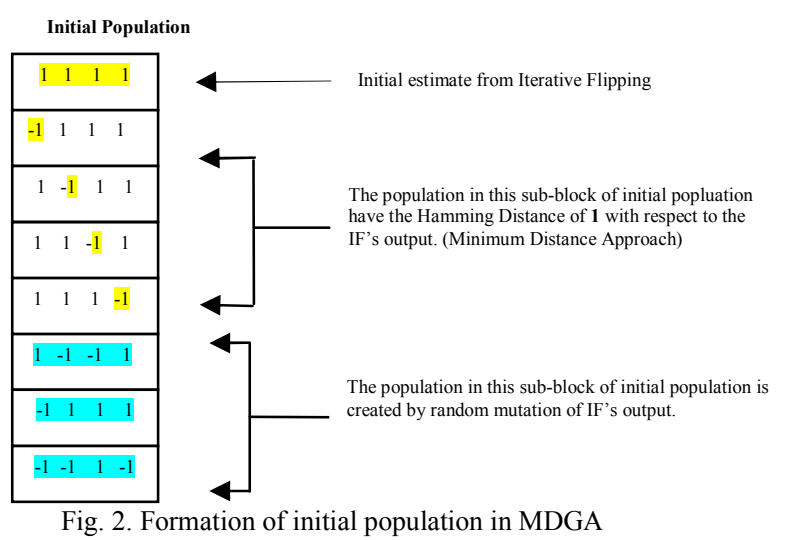

\section{B. Fitness Function}

The fitness function is derived from Equation (7), which is rewritten as

$$
\hat{s_{k}}=\left\{\min \left(\underline{s_{k}}\right)\right\}_{b_{w}}
$$

Each combination of $b_{w}$ is a vector of phase factors, which vector length is equal to the number of sub-blocks. The task of PTS is to measure all the phase factors jointly in order to discover a global minimum $\underline{s_{k}}$ of searching space $\underline{s_{k}}$ by accurately positioning $\hat{b}_{w}$. In order to efficiently reduce PAPR in MC-CDMA system by using PTS technology, we need to find such a combination of vector " $b_{w}$ " that minimizes the above cost function. Hence, GA is invoked in a search for such an optimum solution vector $\hat{b}_{w}$.

\section{Replacement Strategy}

After a successful evaluation of the initial population, each chromosome is ranked according to the fitness from the minimum to the maximum. The truncation selection takes place according to some selection rate, "Xrate" (e.g. Xrate $=1 / 2$ is set in our case initially and to be dynamic further to invoke incest protection). In the beginning of each generation, the " $\left[N_{p} / 2\right\rceil$ " best individuals in the ranking list are selected for the mating, where $\left\lceil N_{p}\right\rceil$ denotes ceiling value of $N_{P}$. Now, considering the population size of 30 , we first select 15 best candidates by the truncation-selection method and then from the two fittest 
parents we form two offspring by performing uniformed cross-over operation. Then, from the next two fittest mating parents we create two more offspring and so on till we form 13 new offspring. We design the mating process in a way that in each generation, the number of offspring generated is equal to $N_{P^{-}}(M+1)$ provided the incest does not appear in the patents candidates.

After successful mating in each generation, we keep the fittest mate remained and generate other $M$ chromosome by performing the same Minimum Distance approach using the fittest mate, i.e. the new pool will contain the fittest mate on the top along with the " $M$ " individuals having Hamming Distance of one between them and the previously formed " $N_{P^{-}}(M+1)$ " number of offspring. This is how we exploit the information of best mate (local search) at each generation as well as we also create a reasonable number of offspring out of the mating pool for exploration.

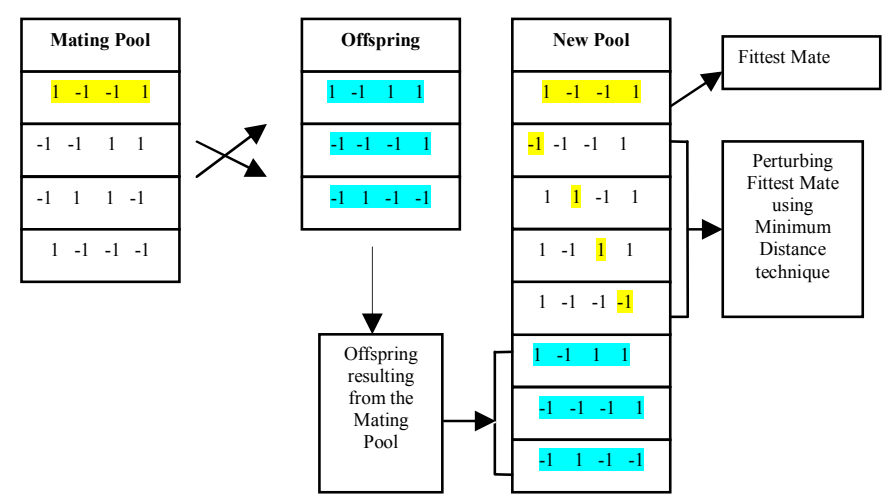

Fig. 3. A Novel Replacement Strategy in MDGA

The MDGA is firstly guided by the output of IF and then in the following generations it is guided by the best mate selected from the mating pool. The fittest-initiated " $M+1$ " individuals along with the " $N_{P^{-}}(M+1)$ " offspring generated from the mating pool ensures that the GA would not likely be stuck at the local maximum. The process involved in the replacement strategy is shown in Fig. 3, with an example population size of 8 chromosomes for a case of four sub-blocks divided in an MC-CDMA system. Note that the schematic crossover point is taken after the $2^{\text {nd }}$ bit.

\section{Type of Mutation \& Crossover}

The new pool undergoes mutation process which further ensures that our GA search would not get stuck around local optimum. Through observing a number of experiments, the constant mutation probability chosen to be 0.1 gives the best performance.

The crossover operator is chosen to be uniformed crossover to increase the diversity of offspring so as to avoid converging at local minima. The crossover probability is chosen to be one. The population size after each generation remains constant reflecting the simplicity of the algorithm in its implementation.

\section{E. Elitism}

The Elitism strategy is invoked to preserve the chromosome of the fittest individual during mutation process. There is only one optimum solution to be found, and hence, the elitism strategy is invoked only for one fittest individual of the total population in each generation. Fig. 4 shows the flow of operation in the proposed GA. In the next section we show the comparison of the PAPR reduction performance and corresponding computational complexity between the proposed MDGA with other schemes.

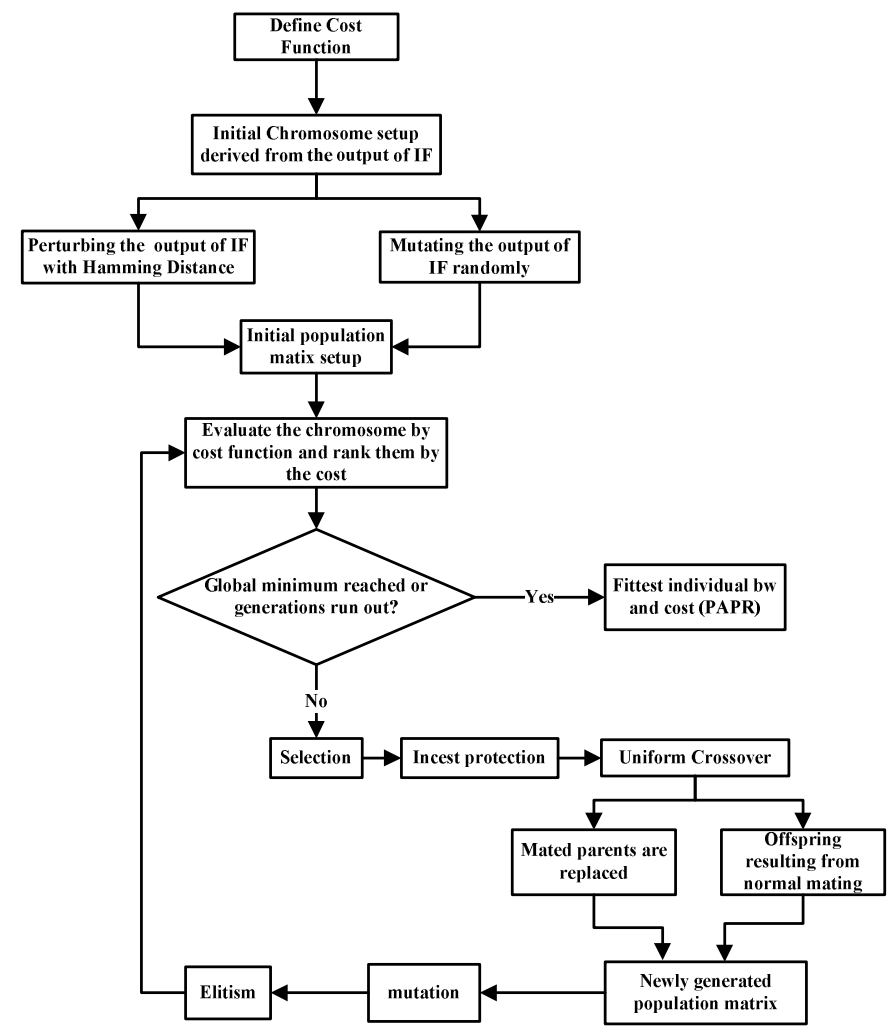

Fig. 4. Flow chart of MDGA-PTS

TABLE I

CONFIGURATION OF THE MDGA-PTS

\begin{tabular}{|c|c|}
\hline SETUP/PARAMETER & METHOD/VALUE \\
\hline Population size $\mathrm{N}_{\mathrm{p}}$ & 30 \\
\hline INITIALIZATION & Initial population setup strategy \\
\hline Selection Method & Truncation Selection \\
\hline Mating pool size & $\leq\left\lceil N_{P} / 2\right\rceil$ depends on the number of \\
& non-identical individuals \\
\hline Crossover operation & Uniformed crossover \\
\hline Crossover Probability $\mathrm{p}_{\mathrm{c}}$ & 1 \\
\hline NEW POOL & New pool created by Hamming Distance \\
scheme
\end{tabular}




\section{Simulation Results}

The complementary cumulative distribution function (CCDF) and computational complexity are the two main measurement criteria to be used. The CCDF represents the probability of PAPR beyond the given threshold, whereas, the computational complexity of the GA, in the context of the total number of cost function evaluations which is defined as the product of the population size and the number of generations. Table I shows the configuration parameters used in the simulations. QPSK modulation type is considered throughout the paper.

In Fig. 5, some results of CCDF versus PAPR are simulated for the MC-CDMA system with 64 subcarriers and QPSK modulation, in which 16 sub-blocks are employed and binary phase factors are used for phase rotation on each sub-block. We choose the iterative flipping (IF) in which the rule of phase rotation adopts the flipping binary phase factor $\{+1,-1\}$ sequentially and conventional GA as comparison. It is noted that the IF method reduces PAPR, but the performance is deteriorated as a sub-optimum. Using a genetic algorithm, the PAPR can be further reduced. However, the computational complexity is still high. The simulation results in Fig. 5 show that the proposed MDGA can provide almost the same performance as exhaustive PTS, but with much lower computational complexity than conventional GA. Based on the same population size, the generations can be reduced at least half.

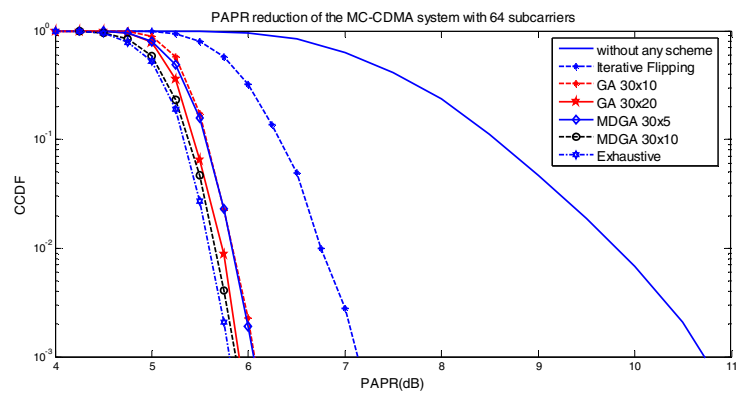

Fig. 5.The CCDF versus PAPR of the MC-CDMA system with 64 subcarriers

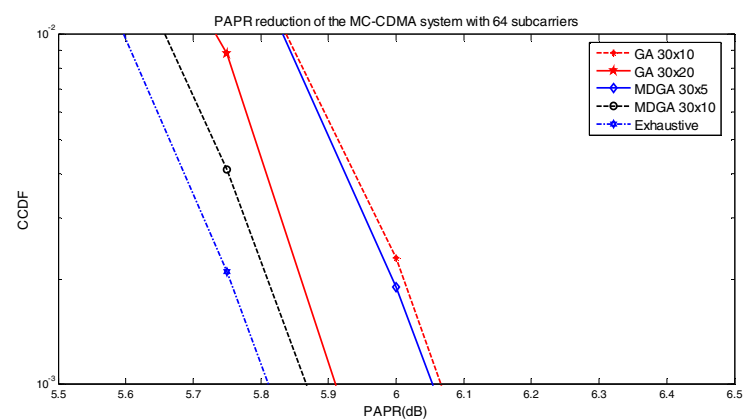

Fig. 6. Zoom-in view of the CCDF versus PAPR of the MC-CDMA with 64 subcarriers

Fig. 6 is the detailed zoom-in version of Fig. 5. It is shown that the performance of MDGA with five generations is even better than that of the conventional GA with ten generations, i.e., $\mathrm{PAPR}=6.05 \mathrm{~dB}$ using $\mathrm{MDGA}$ vs. $\mathrm{PAPR}=6.07 \mathrm{~dB}$ using conventional $\mathrm{GA}$, for $\mathrm{CCDF}=10^{-3}$. Furthermore, there is a significant improvement in PAPR reduction between our MDGA with 10 generations and the traditional GA with 20 generations, i.e. $\mathrm{PAPR}=5.86 \mathrm{~dB}$ with ten generations, as compared to $\mathrm{PAPR}=5.91 \mathrm{~dB}$ with 20 generations.

In Fig. 7, the simulation results are shown for the MC-CDMA system with 128 subcarriers, in which 16 sub-blocks are employed and binary phase factors are used for phase rotation on each sub-block. It is observed that the MDGA can stably perform regardless of an increase of subcarriers. When the $\mathrm{CCDF}=10^{-3}$, the PAPR in Fig. 6 are $6.07 \mathrm{~dB}, 5.91 \mathrm{~dB}$, $6.05 \mathrm{~dB}$ and $5.86 \mathrm{~dB}$ for GA with generations 10, 20 and MDGA with generations 5, 10, respectively. Compared to the PAPR reduction of the MC-CDMA system with 64 subcarriers illustrated in Fig. 6, the corresponding PAPR of CCDF with the value of 0.001 for 128 subcarriers MC-CDMA system in Fig. 8, are $6.74 \mathrm{~dB}, 6.61 \mathrm{~dB}, 6.72 \mathrm{~dB}$ and $6.56 \mathrm{~dB}$ for $\mathrm{GA}$ with generations 10, 20 and MDGA with generations 5, 10, respectively, where Fig. 8 is also the detailed zoom-in version of Fig. 7. Therefore, our proposed MDGA scheme is not affected by an increased number of subcarriers, which means it is a promising candidate for PAPR reduction in a high data rate MC-CDMA system.

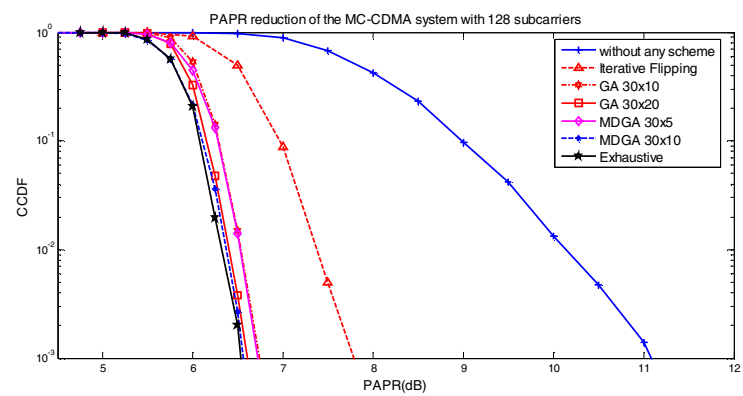

Fig. 7. The CCDF versus PAPR of the MC-CDMA system with 128 subcarriers

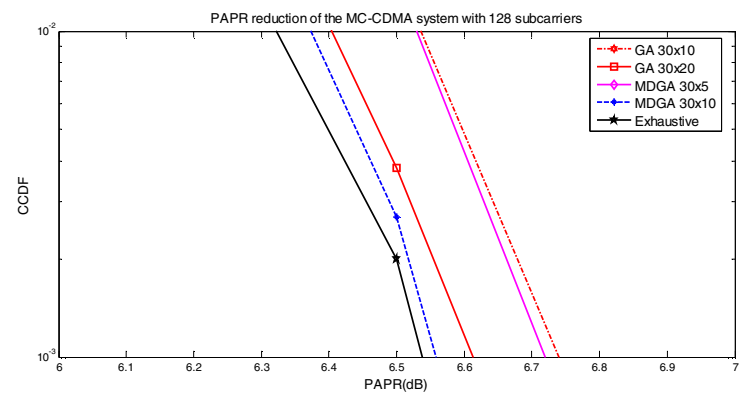

Fig. 8. Zoom-in view of the CCDF versus PAPR of the MC-CDMA with 128 subcarriers

The conventional GA with random searching lacks the guidance from IF's output in each generation and is more likely to deviate the global minima and hence requires increasing the number of generations to refine the optimal solutions before search terminates.

The computational complexity is defined as the number of cost function evaluations needed until reaching the optimum solution [1]. As shown in Table II, MDGA can supply the 
near-optimum solution within only $30 \times 10+16=316$ cost function evaluations $(\mathrm{Gen}=10$, Pop $=30)$ as compared to $2^{16}$ $(65,536)$ evaluations taken by exhaustive PTS search. Hence, it achieves $99.52 \%$ reduction in complexity as compared to original exhaustive PTS based PAPR reduction. Also from Fig. 5 , as compared to conventional GA with $600 \quad(\mathrm{Pop}=30$, $\mathrm{Gen}=20$ ) cost function evaluations, the MDGA reaches the optimum in only $316(\mathrm{Pop}=30$, Gen=10) cost function evaluations, ensuring $47.3 \%$ complexity reduction in terms of cost function evaluations. Note, as the generations increase, the complexity reduction can be further improved as $50+\%$.

TABLE II

COMPARISON OF COMPUTATIONAL COMPLEXITY AMONG DIFFERENT SCHEMES BASED ON 64 SUBCARRIERS OF THE MC-CDMA SYSTEM

EX: EXhaustive PTS, IF: ITERATIVE FLipPing, GA: ConventionAl GeNETIC Algorithm, MDGA: Minimum Distance GUided Genetic Algorithm

\begin{tabular}{|c|c|c|}
\hline SCHEMES & $\begin{array}{c}\text { COMPUTATIONAL } \\
\text { COMPLEXITY }\end{array}$ & $\begin{array}{c}\text { PAPR } \\
(\mathrm{CCDF}=0.001)\end{array}$ \\
\hline $\mathrm{EX}$ & $\mathrm{W}^{\mathrm{M}=}=65536(\mathrm{~W}=2, \mathrm{M}=16)$ & $5.81 \mathrm{~dB}$ \\
\hline $\mathrm{IF}$ & $(\mathrm{W}-1) \times \mathrm{M}=16(\mathrm{~W}=2, \mathrm{M}=16)$ & $7.12 \mathrm{~dB}$ \\
\hline $\mathrm{GA}$ & $\mathrm{P} \times \mathrm{G}=600(\mathrm{P}=30, \mathrm{G}=20)$ & $5.91 \mathrm{~dB}$ \\
\hline$M D G A$ & $\begin{array}{l}\mathrm{P} \times \mathrm{G}+(\mathrm{W}-1) \times \mathrm{M}=316 \\
(\mathrm{P}=30, \mathrm{G}=10, \mathrm{~W}=2, \mathrm{M}=16)\end{array}$ & $5.86 \mathrm{~dB}$ \\
\hline
\end{tabular}

\section{CONCLUSIONS}

PTS is deemed as an efficient method to reduce PAPR in multi-carrier system such as a MC-CDMA system regardless the number of subcarriers. However, the fatal weakness of such technology is its inherent tremendous computational complexity issue. In this paper, searching phase factors of PTS is fashioned as a particular multi-objects combinatory optimization problem and a novel MDGA algorithm is proposed to significantly mitigate the computational complexity of PTS as well as acquiring the optimal results. Our scheme carried out a balanced search based on an equal amount of exploration and exploitation within each step of the GA. The Minimum Distance technique is used effectively along with a novel replacement strategy. We have shown a significant improvement in PAPR reduction performance while reducing dramatically computational complexity as compared to exhaustive PTS searching and traditional GA assisted PTS. The simulation results also show that the superiority of our scheme holds with various numbers of subcarriers.

\section{REFERENCES}

[1] L. Hanzo, L.-L. Yang, E.-L. Kuan, and K. Yen, "Single- and Multi-Carrier CDMA: Multi-User Detection, Space-Time Spreading, Synchronization, Standards and Networking." Piscataway, NJ: IEEE Press/Wiley, 2003.

[2] R. Prasad; S. Hara, "Multicarrier Techniques For 4G Mobile Communications, "IEEE Press/Wiley, June 2003

[3] S. Hara and R. Prasad, "Overview of Multi-Carrier CDMA," IEEE Communications Magazine, vol. 35, pp. 126-133, Dec. 1997.
[4] S.-K. Deng and M.-C. Lin, "OFDM PAPR Reduction Using Clipping with Distortion Control," IEEE International Conference on Communications, vol.4, pp. 2563-2567, May 2005.

[5] N. N. Malik, A. Nik, N. Ngajikin, S. M. Idrus and N. D. A. Latif, "Peak to Average Power Ratio (PAPR) Reduction in OFDM System,"

International RF and Microwave Conference (RMC), pp. 75-79, 12-14 Sept. 2006.

[6] O. Masaki and H. Takeshi, "PAPR Reduction Method Using Clipping and Peak-Windowing in CI/OFDM System, "IEEE Vehicular Technology Conference (VTC 2007), pp.1356-1360, 30 Sept.- 3 Oct. 2007.

[7] R. D. J. Van Nee, "OFDM Codes for Peak-to-Average Power Reduction and Error Correction, " IEEE Global Telecommunications Conference, London, pp. 740-744, 18-22, Nov. 1996.

[8] L. J. Cimini. Jr. and N.R. Sollenberger. "Peak-to-Average Power Ratio Reduction of an OFDM Signal using Partial Transmit Sequences, " IEEE International Conference on Communications. (ICC'99), vol. 1, pp. 511-515, 6-10 June. 1999

[9] N. Ohkubo and T. Ohtsuki, “ A Peak to Average Power Ratio Reduction of Multicarrier CDMA Using Selected Mapping," Vehicular Technology Conference, Proceedings. (VTC), vol.4 pp.2086-2090, 24-28 Sept. 2002.

[10] S. H. Muller and J. B. Huber, "OFDM with Reduced Peak-to-Average Power Ratio by Optimum Combination of Partial Transmit Sequences, " Electronics Letters, vol.33 (5), pp. 368-369, Feb. 1997

[11] W. S. Ho, A. Madhukumar, and F. Chin, "Peak-to-Average Power Reduction Using Partial Transmit Sequences: A Suboptimal Approach Based on Dual Layered Phase Sequencing," IEEE Trans. Broadcasting, vol. 49, no. 2, pp. 225-231, June 2003.

[12] Y.-H. You, W.-G. Jeon, J.-H. Paik, and H.-K. Song, “A Simple Construction of OFDM-CDMA Signals With Low Peak-to-Average Power Ratio, " IEEE Trans. Broadcasting, vol. 49, no. 4, pp. 403-407, Dec. 2003.

[13] O.-J. Kwon and Y.-H. Ha, "Multi-Carrier Pap Reduction Method Using Sub-Optimal PTS with Threshold," IEEE Trans. Broadcasting, vol. 49, no.2, pp. 232-236, June 2003.

[14] D.-W. Lim, S.-J. Heo, J.-S. No, and H. Chung, “A New PTS OFDM Scheme with Low Complexity for PAPR Reduction," IEEE Trans. Broadcasting, vol. 52, no. 1, pp. 77-82, Mar. 2006.

[15] L. Yang, R. S. Chen, Y. M. Siu, and K. K. Soo, "PAPR Reduction of An OFDM Signal by Use of PTS with Low Computational Complexity," IEEE Trans. Broadcasting, vol. 52, no. 1, pp. 83-86, Mar. 2006.

[16] J. H. Holland, "Adaptation in Natural and Artificial Systems," University of Michigan Press, Ann Arbor, 1975

[17] C. Tellambura, "Phase Optimization Criterion for Reducing Peak-to-Average Power Ratio in OFDM, "Electronics Letters, vol. 34 (2), pp. 169-170, 22 Jan. 1998 